INPLASY

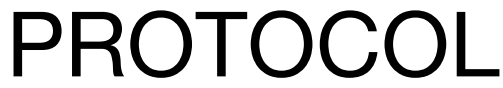

To cite: Htet et al. The effect of metformin on gastric cancer in patients with type 2 diabetes mellitus: an umbrella review. Inplasy protocol 202130110. doi:

10.37766/inplasy2021.3.0110

Received: 30 March 2021

Published: 30 March 2021

Corresponding author:

Cho Naing

cho3699@gmail.com

Author Affiliation:

International Medical

University ( IMU)

Support: IMU (R221/2018.

Review Stage at time of this submission: Piloting of the study selection process.

\section{The effect of metformin on gastric cancer in patients with type 2 diabetes mellitus: an umbrella review}

Htet, $\mathrm{NH}^{1}$; Naing, C2; Tung, WS3; Mak, JW4.

Review question / Objective: To evaluate the strength and validity of evidence for the association between metformin and the risk of gastric cancer in patients with type 2 diabetes mellitus.

Condition being studied: Gastric cancer patients with type 2 diabetes mellitus.

Study designs to be included: Retrospective or prospective cohort studies, case-control studies, randomised controlled trials will be included, if they compared the effect on gastric cancer between those who used metformin with those who did not (or those who used other antidiabetic drugs).

INPLASY registration number: This protocol was registered with the International Platform of Registered Systematic Review and Meta-Analysis Protocols (INPLASY) on 30 March 2021 and was last updated on 30 March 2021 (registration number INPLASY202130110).

Conflicts of interest:

None declared.

\section{INTRODUCTION}

Review question / Objective: To evaluate the strength and validity of evidence for the association between metformin and the risk of gastric cancer in patients with type 2 diabetes mellitus.

Rationale: Gastric cancer (ICD-10-CM Code $\mathrm{C}_{16.2)}$ is a multi-factorial disease in its pathology. Diabetes is a chronic, metabolic disease characterized by elevated blood glucose levels. The most common is type 2 diabetes (T2DM), usually in adults. Epidemiological studies reported that T2DM patients have a significant increase in cancer risk and mortality compared to non-diabetic patients. Metformin (a biguanide) is widely used to treat diabetes mellitus and is reported to confer preventive and survival benefits for various malignancies including gastric 
cancer. There is a growing number of metaanalyses in this field, but with different conclusions. Hence, it is necessary to examine the diversity present in the systematic reviews of the association between gastric cancer and metformin. For instance, the duration of treatment with metformin, the level of control (e.g. HbA1c, reduction in inflammatory biomarkers, reduction in BMI etc.) were important variables that might be confounded in the effect estimates. An umbrella review can address a broad scope related to a topic of interest ideal suited to present a clear evidence related to a particular question.

Condition being studied: Gastric cancer patients with type 2 diabetes mellitus.

\section{METHODS}

Search strategy: The simple search strategy will be included: ("metformin" OR "metformin hydrochloride") AND ("gastric cancer" OR "gastric neoplasm" OR "stomach neoplasm") AND ("systematic review" OR "meta-analysis").

Participant or population: Patients with gastric cancer, taking treatment for diabetes mellitus.

Intervention: Metformin for treatment of diabetes.

Comparator: Alternative antidiabetic drugs or no drug treatment for diabetes mellitus.

Study designs to be included: Retrospective or prospective cohort studies, case-control studies, randomised controlled trials will be included, if they compared the effect on gastric cancer between those who used metformin with those who did not (or those who used other antidiabetic drugs).

Eligibility criteria: Systematic reviews (with or without meta-analyses) that fullfil the above mentioned criteria in the PICO/PECO format framework will be eligible.

Information sources: PubMed, Cochrane library and Google Scholars.
Main outcome(s): Survival effect on gastric cancer measured with hazard ratio (HR) and corresponding $95 \%$ confidence interval (Cl).

Quality assessment / Risk of bias analysis: The methodological quality/risk of bias of included systematic reviews will be assessed with the use of AMSTAR-2 tool (a measurement tool to assess systematic reviews). (Shea, 2017).

Strategy of data synthesis: As described elsewhere (Fusar-Poli, 2018; Pollock, 2021), the following step-wise approaches will be performed. i. Estimation of summary effect with both fixed-effect and random-effects models. We will rely on the analyses reported in the included systematic reviews as much as possible. ii. Assessment of heterogeneity iii. Estimation of prediction interval: iv. Evaluation of small study effects v. Evaluation of excess significance. vi. Stratification of evidence.

Subgroup analysis: If data permitted, systematic reviews including more than/ less than 10 individual studies.

Sensitivity analysis: If data permitted, excluding systematic reviews with HR solely derived from univariate analysis.

Language: English.

Country(ies) involved: Malaysia.

Keywords: Gastric cancer; Diabetes mellitus; Metformin; Umbrella review.

Contributions of each author:

Author 1 - Norah Htet Htet.

Author 2 - Cho Naing.

Author 3 - Wong Siew Tung.

Author 4 - Joon Wah Mak. 